\title{
Neural Machine Translation of Logographic Languages Using Sub-character Level Information
}

\author{
Longtu Zhang \\ Tokyo Metropolitan University \\ 6-6 Asahigaoka, Hino, \\ Tokyo 191-0065, Japan \\ zhang-longtu@ed.tmu.ac.jp
}

\author{
Mamoru Komachi \\ Tokyo Metropolitan University \\ 6-6 Asahigaoka, Hino, \\ Tokyo 191-0065, Japan \\ komachi@tmu.ac.jp
}

\begin{abstract}
Recent neural machine translation (NMT) systems have been greatly improved by encoder-decoder models with attention mechanisms and sub-word units. However, important differences between languages with logographic and alphabetic writing systems have long been overlooked. This study focuses on these differences and uses a simple approach to improve the performance of NMT systems utilizing decomposed sub-character level information for logographic languages. Our results indicate that our approach not only improves the translation capabilities of NMT systems between Chinese and English, but also further improves NMT systems between Chinese and Japanese, because it utilizes the shared information brought by similar sub-character units.
\end{abstract}

\section{Introduction}

Neural machine translation (Cho et al., 2014) (NMT) systems based on sequence-to-sequence models (Sutskever et al., 2014) have recently become the de facto standard architecture. The models use attention mechanisms (Bahdanau et al., 2015; Luong et al., 2015) to keep records of all encoding results, and can focus on particular parts of these results during decoding, so that the model can produce longer and more accurate translations. Sub-word units are another technique first introduced by Sennrich's (2016) application of the byte pair encoding (BPE) algorithm, and are used to break up words in both source and target sentences into sequences of smaller units, learned without supervision. This alleviates the risk of producing <unk > symbols when the model encounters infrequent "unknown" words, also known as the out-of-vocabulary (OOV) problem. Moreover, sub-word units, which can be viewed as learned stems and affixes, can help the NMT model better encode the source sentence and decode the target sentence, particularly when the source and target languages share some similarities.

Almost all of the methods used to improve NMT systems were developed for alphabetic languages such as English, French, and German as either the source or target language, or both. An alphabetic language typically uses an alphabet: a small set of letters (basic writing symbols) that each roughly represents a phoneme in the spoken language. Words are composed by ordered letters, and sentences are composed by spacesegmented ordered words. However, in other major writing systems - namely, logographic (or character-based) languages such as Chinese, Japanese, and traditional Korean-strokes are used to construct ideographs; ideographs are used to construct characters, which are the basic units for meaningful words. Words can then further compose sentences. In alphabetic languages, sub-word units are easy to identify, whereas in logographic languages, a similar effect can be achieved only if sub-character level information is taken into consideration. ${ }^{1}$

Having noticed this significant difference between these two writing systems, Shi et al. (2015), Liu et al. (2017), Peng et al. (2017), and Cao et al. (2017) used stroke-level information for logographic languages when constructing word embeddings; Toyama et al. (2017) used visual information for strokes and Japanese Kanji

\footnotetext{
${ }^{1}$ Taking the ASPEC corpus as an example, the average word lengths are roughly 1.5 characters (Chinese words, tokenized by Jieba tokenizer), 1.7 characters (Japanese words, tokenized by MeCab tokenizer), and 5.7 characters (English words, tokenized by Moses tokenizer), respectively. Therefore, when a sub-word model of similar vocabulary size is applied directly, English sub-words usually contain several letters, which are more effective in facilitating NMT, whereas Chinese and Japanese sub-words are largely just characters.
} 
radicals in a text classification task. ${ }^{2}$

Some studies have performed NMT tasks using various sub-word "equivalents". For instance, Du and Way (2017) trained factored NMT models using "Pinyin" 3 sequences on the source side. Unfortunately, they did not apply a BPE algorithm during training, and their model also cannot perform factored decoding. Wang et al. (2017) directly applied a BPE algorithm to character sequences before building NMT models. However, they did not take advantage of sub-character level information during the training of sub-word and NMT models. Kuang and Han (2018) also attempted to use a factored encoder for Chinese NMT systems using radical data. It is worth noting that although the idea of using ideographs and strokes in NLP tasks (particularly in NMT tasks) is not new, no previous NMT research has focused on the decoding process. If it is also possible to construct an ideograph/stroke decoder, we can further investigate translations between logographic languages. Additionally, no NMT research has previously used stroke data.

To summarize, there are three potential information gaps associated with current studies on NMT systems for logographic languages using sub-character level data: 1) no research has been performed on the decoding process; 2) no studies have trained models using sub-character level sub-words; and 3) no studies have attempted to build NMT models for logographic language pairs, despite their sharing many similarities. This study investigates whether sub-character information can facilitate both encoding and decoding in NMT systems and between logographic language pairs, and aims to determine the best sub-character unit granularity for each setting.

The main contributions of this study are threefold:

1. We create a sub-character database of Chinese character-based languages, and conduct MT experiments using various types of subcharacter NMT models.

\footnotetext{
${ }^{2}$ To be more precise, there is another so-called syllabic writing system, which uses individual symbols to represent symbols rather than phonemes. Japanese hiragana and katakana are actually syllabic symbols rather than ideographs. In this paper, we focus only on the logographic part.

${ }^{3}$ An official Romanization system for standard Chinese in mainland China. Pinyin includes both letters and diacritics, which represent phonemic and tonal information, respectively.
}

2. We facilitate the encoding or decoding process by using sub-character sequences on either the source or target side of the NMT system. This will improve translation performance; if sub-character information is shared between the encoder and decoder, it will further benefit the NMT system.

3. Specifically, Chinese ideograph ${ }^{4}$ data and Japanese stroke data are the best choices for relevant NMT tasks.

\section{Background}

\subsection{NMT with Attention Mechanisms and Sub-word Units}

In this study, we applied a sequence-to-sequence model with an attention mechanism (Bahdanau et al., 2015). The basic recurrent unit is the "long short-term memory" (Hochreiter and Schmidhuber, 1997) unit. Because of the nature of the sequence-to-sequence model, the vocabulary size must be limited for the computational efficiency of the Softmax function. In such cases, the decoder outputs an <unk> symbol for any word that is not in the vocabulary, which will harm the translation quality. This is called the out-ofvocabulary (OOV) problem.

Sub-word unit algorithms (such as BPE algorithms) first break up a sentence into the smallest possible units. Then, two adjacent units at a time are merged according to some standard (e.g., the co-occurrence frequency). Finally, after $n$ steps, the algorithm collects the merged units as "subword" units. By using sub-word units, it is possible to represent a large number of words with a small vocabulary. Originally, sub-word units were only applied to unknown words (Sennrich et al., 2016). However, in the recent GNMT (Wu et al., 2016) and transformer systems (Vaswani et al., 2017), all words are broken up into subword units to better represent the shared information.

For alphabetic languages, researchers have indicated that sub-word units are useful for solving OOV problems, and that shared information can further improve translation quality. The Sentencepiece project ${ }^{5}$ compared several combinations of word-pieces (Kudo, 2018) and BPE sub-word

\footnotetext{
${ }^{4}$ We use the term "logographic" to refer to writing systems such as Chinese characters and Japanese Kanji, and "ideograph" to refer to the character components.

${ }^{5} \mathrm{https}$ //github.com/google/sentencepiece
} 


\begin{tabular}{|c|c|c|c|}
\hline Character & $\begin{array}{l}\text { Semantic } \\
\text { ideograph }\end{array}$ & $\begin{array}{l}\text { Phonetic } \\
\text { ideograph }\end{array}$ & Pinyin \\
\hline 驰 run & 马 horse & 也 & chH \\
\hline 池 pool & 水( $(\dot{y})$ water & 也 & chH \\
\hline 施 impose & 方 direction & 也 & sh \\
\hline 弛 loosen & 弓 bow & 也 & chH \\
\hline 地 land & 土 soil & 也 & $\mathrm{dM}$ \\
\hline 驱 drive & 马 horse & 区 & $\mathrm{q}$ \\
\hline
\end{tabular}

Table 1: Examples of decomposed ideographs of Chinese characters. The composing ideographs of different functionality might be shared across different characters.

models in English/Japanese NMT tasks. The subword units were trained on character (Japanese Kanji and Hiragana/Katakana) sequences. Similarly, Wang et al. (2017) attempted to compare the effects of different segmentation methods on NMT tasks, including "BPE" units trained on Chinese character sequences.

\subsection{Sub-character Units in NLP}

In alphabetic languages, the smallest unit for sub-word unit training is the letter; in characterbased languages, the smallest units should be subcharacter units, such as ideographs or strokes. Because sub-character units are shared across different characters and have similar meanings, it is possible to build a significantly smaller vocabulary to cover a large amount of training data. This has been researched quite extensively within tasks such as word embeddings, as mentioned previously.

As we can see from the examples in Table 1, there are several independent Chinese characters. Each character can be split into at least two ideographs: a semantic ideograph and a phonetic ideograph. ${ }^{6}$ More importantly, the same ideograph can be shared by different characters denoting similar meanings. For example, the first five characters (驰, 池, 施, 弛 and 地) have similar pronunciation (and they are written similarly in Pinyin) because they share the same phonetic ideograph “也”. Similarly, semantic ideographs can be shared across characters and denote a similar semantic meaning. For example, the first character "驰" and the last character "马区" share same semantic ideograph "马" (meaning "horse"); and their semantic meanings are closely related ("run" and "drive", respec-

\footnotetext{
${ }^{6}$ Semantic ideographs denote the meaning of a character, whereas phonetic ideographs denote the pronunciation.
}

\begin{tabular}{ccc}
\hline Word & Meaning & Ideographs \\
\hline 树木 & Wood & 木对木 \\
森林 & Forest & 木木木木木 \\
\hline
\end{tabular}

Table 2: Examples of multi-character words in Chinese and their ideograph sequences.

tively). A few ideographs can also be treated as standalone characters.

To the best of our knowledge, however, no research has been performed on logographic language NMT beyond character-level data, except in the work of Du and Way (2017), who attempted to use Pinyin sequences instead of character sequences in Chinese-English NMT tasks. Considering the fact that there are a large number of homophones and homonyms in Chinese languages, it was difficult for this method to be used to reconstruct characters in the decoding step.

\section{NMT Using Sub-character Level Units}

\subsection{Ideograph Information}

When building NMT vocabulary, the use of subcharacters (instead of words, characters, and character level sub-words) can greatly condense vocabulary size. For example, a vocabulary can be decreased from 6,000 to 10,000 character types ${ }^{7}$ to hundreds ${ }^{8}$ of ideographs. Table 2 presents two Chinese words composed of four different characters that have very close meanings. Characterbased NMT models treat these characters separately as one-hot vectors. In contrast, if the two words are broken down into ideograph sequences, they overlap significantly. Then, only two ideographs are needed to compose the vocabulary of the two words. The computational load will be reduced, and the chances of training neurons responsible for low-frequency vocabularies will increase.

Moreover, sub-character units can serve as building blocks for constructing characters that are not present in the training data, because all CJK characters are designed to be composed of a limited number of ideographs in UNICODE standards.

\subsection{Stroke Information}

All ideographs can be further decomposed into strokes, which are smaller units and have an even

\footnotetext{
${ }^{7}$ According to the ASPEC corpus.

${ }^{8} 214$ as defined in UNICODE 10.0 standard and 517 as defined in CNS11643 charset.
} 
smaller number of types. Therefore, we also propose training our model on stroke sequences. There are five basic stroke types for Chinese characters and Japanese Kanji: "horizontal" (一), "vertical" ( | ), "right falling" ( $\smile$ ), "left falling" ( ) ), and "break" ( ). Each stroke type can be further sub-categorized into several stroke variations. For example, left falling strokes contain both long and short left fallings ( $($ and $ノ)$, while a break contains many more variations, such as ᄂ, ᄂ, ᄀ, and つ (details can be found in Appendix A).

In practice, the CNS11643 charset $^{9}$ is used to transform each character into a stroke sequence, where unfortunately only "stroke-type" information is available. In this study, we manually transcribed all ideographs into stroke sequences using 33 pre-defined strokes.

\subsection{Character Decomposition}

The CNS11643 charset is used to facilitate character decomposition, where Chinese, Japanese, and Korean characters are merged into a single character type based on similarities in their forms and meanings. This is potentially beneficial; for example, if Chinese and Japanese vocabularies are built, they will authentically share some common types. There are 517 so-called "components" (i.e., ideographs) pre-defined in CNS11643. This ensures that all characters can be divided into certain sequences of components. For example, the character “可” can be split into “丁” and “口”; and the character “君” can be split into “尹” and “口”. Details can be found on the CNS11643 website ${ }^{10}$. Using this ideograph decomposition information, all Chinese and Japanese sentence data can be transformed into new ideograph sequences; then, using the manually transcribed stroke decomposition data introduced in Section 3.2, we can also obtain new stroke sequences.

Note that although there are no clear indications of how the components/strokes are structured together, the sequence potentially contains structural information, because the writing of characters always follows a certain order, such as "up-down", "outside-in", etc. We also note that UNICODE 10.0 has introduced symbols indi-

\footnotetext{
${ }^{9}$ The CNS1 1643 charset is published and maintained by the Taiwan government.

http://www.cns11643.gov.tw/AIDB/welcome_en.do

${ }^{10} \mathrm{http}: / / \mathrm{www} . \mathrm{cns} 11643$. gov.tw/search.jsp?ID $=13$
}

\begin{tabular}{|c|c|}
\hline Language & Word \\
\hline JP-character & 風 景 \\
\hline JP-ideograph & 几一虫 日十口小_1 \\
\hline JP-stroke & 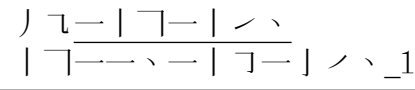 \\
\hline $\mathrm{CN}$-character & 风 景 \\
\hline $\mathrm{CN}$-ideograph & 几メ 日ナ口小_1 \\
\hline $\mathrm{CN}$-stroke & 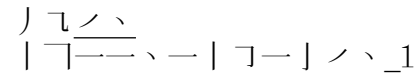 \\
\hline EN & landscape \\
\hline
\end{tabular}

Table 3: The Japanese word 風景 and Chinese word 风景 both mean “landscape" in English, and they only differ in the middle part of the first character. Note that there are "_ 1" tags at the ends of some decomposed sequences to distinguish between possible duplications.

cating sub-character structures (Ideographic Description Characters), which provide a clearer indication of character compositions. We will make further use of this information in future studies.

To ensure that there are no duplicated ideograph and stroke sequences for different characters and multi-character words, we post-tag the sequences on the duplicated ones using "_1", "_2", etc. Table 3 shows an example of character decomposition in Chinese and Japanese ${ }^{11}$.

\section{Experiments on Chinese-Japanese-English Translation}

To answer our research questions, we set up a series of experiments to compare NMT models of logographic languages trained on word sequences, character-level sub-word unit sequences, and ideograph- and stroke-level subword unit sequences.

We performed two lines of experiments:

1. We trained NMT models between logographic language and alphabetic language combinations, i.e., Japanese/Chinese and English. In each model, we varied the data granularity for the logographic language, using "character level" or "sub-character level" (ideograph level and stroke level) granularities. We used the character level

\footnotetext{
${ }^{11}$ For example, the ideograph and stroke sequences for character 景 are the same as those for character 晾 (meaning "to dry in the sun"). However, these two characters have different architectures ("top-down" vs. "left-right"). Post-tags are thus appended in order to distinguish them. Similarly, characters 风 and 凤 have the same ideograph and stroke sequences, and thus must be post-tagged.
} 
NMT models as our baselines, and investigated whether the sub-character level NMT models could outperform the baseline models.

2. We trained NMT models between combinations of two logographic languages, i.e., Chinese and Japanese. Similarly, we used data sets with different granularities: 1) Models lacking sub-character level data. 2) Models having sub-character level data on both sides (to confirm the results of the previous experiment). For the experiments, the models will have both source and target sides. The models will use sub-character level data with/without shared vocabularies (namely, ideograph models, stroke models, ideograph-stroke models, stroke-ideograph models, and ideograph/stroke models with shared vocabularies). 3) Pinyin baselines according to (Du and Way, 2017), where both Pinyin word sequences with tones and character sequences with Pinyin factors are used with the encoder.

\subsection{Dataset}

We trained our baselines and experiments using Chinese, Japanese, and English. The Asian Scientific Paper Excerpt Corpus (ASPEC (Nakazawa et al., 2016)) and Casia2015 ${ }^{12}$ corpus were used for this purpose.

ASPEC contains a Japanese-English paper abstract corpus of 3 million parallel sentences (ASPEC-JE) and a Japanese-Chinese paper excerpt corpus of 680,000 parallel sentences (ASPEC-JC). We used the first million confidently aligned parallel sentences in ASPECJE and all of the ASPEC-JC data to cover Japanese-English and Japanese-Chinese language pairs. The Casia2015 corpus contains approximately 1 million parallel Chinese-English sentences. All data in the Casia2015 corpus were used to cover Chinese-English language pairs. During training, the maximum length hyperparameter was adjusted to ensure $90 \%$ coverage of the training data. For development and testing, the ASPEC corpus has an official split between the development set and test set; however, because the Casia 2015 corpus is not similarly split,

\footnotetext{
${ }^{12} \mathrm{http} / / / \mathrm{nlp}$.nju.edu.cn/cwmt-wmt/, provided by the Institute of Automation, Chinese Academy of Sciences.
}

we made random selections from the development set and test set of 1,000 sentences each.

\subsection{Settings}

Different pre-tokenization methods were applied to the data in three languages (if applicable). A Moses tokenizer was applied to the English data; a Jieba ${ }^{13}$ tokenizer using the default dictionary was applied to the Chinese data; and a MeCab ${ }^{14}$ tokenizer using the IPA dictionary was applied to the Japanese data. For the Pinyin baseline, the pypinyin ${ }^{15}$ Python library was used to transcribe the Chinese character sequence into a Pinyin sequence.

In both of the experiment lines discussed above, data at the "word", "character", "ideograph", and "stroke" levels were used in combinations. For "word" level data, only dictionarybased segmentation was applied; for the other three levels of data, the byte pair encoding (BPE) models were trained and applied, with a vocabulary size of 8,000. In the second line of experiments, where both the source and target sides were logographic languages, we added "character" level data without BPE ("char") for comparison. Additionally, shared vocabularies were applied when both the source and target had the same data granularity level (meaning that both the source and target side would have the same vocabulary $)^{16}$.

A basic RNNsearch model (Bahdanau et al., 2015) with two layers of long short-term memory (LSTM) units was used. The hidden size was 512. A normalized Bahdanau attention mechanism was applied at the output layer of the decoder. We developed our model based on TensorFlow ${ }^{17}$ and its neural machine translation tutorial ${ }^{18}$.

The model was trained on a single GeForce GTX TITAN X GPU. During training, the SGD optimizer was used, and the learning rate was set at 1.0. The size of the training batch was set to 128 , and the total global training step was 250,000 . We also decayed the learning rate as the training progressed: after two-thirds of the train-

\footnotetext{
${ }^{13} \mathrm{https} / / /$ github.com/fxsjy/jieba

${ }^{14} \mathrm{http}: / /$ taku910.github.io/mecab/

${ }^{15} \mathrm{https} / / /$ github.com/mozillazg/python-pinyin

${ }^{16}$ The shared vocabulary can be trained by a BPE model on a concatenated corpus of source and target sentences.

${ }^{17} \mathrm{https}$ //github.com/tensorflow

${ }^{18} \mathrm{https}: / /$ github.com/tensorflow/nmt
} 


\begin{tabular}{lcc}
\hline \multicolumn{2}{c}{ English-Japanese NMT } & BLEU \\
\hline EN_word & JP_word & 36.1 \\
EN_word & JP_character & 38.3 \\
EN_word & JP_ideograph & $40.3^{*}$ \\
EN_word & JP_stroke & $\mathbf{4 1 . 3}^{*}$ \\
\hline \multicolumn{2}{c}{ Japanese-English NMT } & BLEU \\
\hline JP_word & EN_word & 25.5 \\
JP_character & EN_word & 26.3 \\
JP_ideograph & EN_word & $26.8^{*}$ \\
JP_stroke & EN_word & $\mathbf{2 7 . 0 ^ { * }}$ \\
\hline \hline \multicolumn{2}{c}{ English-Chinese NMT } & BLEU \\
\hline EN_word & CN_word & 11.8 \\
EN_word & CN_character & 10.3 \\
EN_word & CN_ideograph & $\mathbf{1 4 . 6}$ \\
EN_word & CN_stroke & $14.1^{*}$ \\
\hline \multicolumn{2}{c}{ Chinese-English NMT } & BLEU \\
\hline CN_word & EN_word & 14.7 \\
CN_character & EN_word & 14.5 \\
CN_ideograph & EN_word & $\mathbf{1 5 . 6 *}$ \\
CN_stroke & EN_word & $15.5^{*}$ \\
\hline
\end{tabular}

Table 4: Experimental results (BLEU scores) of NMT systems for Japanese/English and Chinese/English language pairs. All the scores are statistically significant at $p=0.0001$ (marked by $*$ ).

ing steps, we set the learning rate to be four times smaller until the end of training. Additionally, we set the drop-out rate to 0.2 during training.

BLEU was used as the evaluation metric in our experiments. For Chinese and Japanese data, a KyTea tokenization was applied before we applied BLEU, following the WAT (Workshop on Asian Translation) leaderboard standard. To validate the significance of our results, we ran bootstrap re-sampling (Koehn, 2004) for all results using Travatar (Neubig, 2013) at a significance level of $p=0.0001$.

\subsection{Results}

\subsubsection{NMT of Logographic and Alphabetic Language Pairs}

Table 4 shows the experimental results for the Japanese/English and Chinese/English language pairs in both translation directions. Generally, for each of the experiment settings, the models using ideograph and stroke data outperformed the baseline systems, regardless of the language pair or translation direction. However, for the Japanese/English language pair, the stroke sequence models performed better. For the Chinese/English language pairs, the ideograph sequence models worked better. The reason for these differences will be discussed in detail in
Section 5.

\subsubsection{NMT of Logographic Language Pairs}

Table 5 shows the results for all baselines and proposed models. Among the character-level baselines, the "char" models and "bpe" models outperformed the "word" models in both translation directions. When we applied a shared vocabulary to the "bpe" models, the models achieved the best BLEU scores in both translation directions. These character-level baselines conform with previous studies indicating that sub-word units improve the performance of NMT systems, and that whenever both the source and target side data have similarities in their writing systems, shared vocabularies will further enhance performance.

Sub-character level models aim to replicate similar results to those presented in Section 4.3.1, because only one side of these models uses subcharacter level data. For Japanese-Chinese translation directions, half of the models showed a significant improvement over the baselines, whereas for Chinese-Japanese translation directions, five out of six models showed significant improvements.

When both the source and target side used the same sub-character level data (either ideograph or stroke data), the experimental results also showed significant improvement over character baselines. Additionally, the ideograph models outperformed stroke models. When shared vocabularies were applied to the models, the ideograph models exhibited slight performance improvements $(0.1 \sim$ 0.4 BLEU point), and the stroke models exhibited dramatically decreased performance $(0.9 \sim$ 1.1 BLEU points). However, no model here outperformed the sub-character baselines.

To further exploit the power of sub-character units, the last models having different levels of sub-character units on the source and target side were trained. The results conform with what we found in Section 4.3.1: the models using Chinese ideograph data and Japanese stroke data exhibited the best performance, regardless of whether they were applied at the source or target side. For Japanese-Chinese translations, the best BLEU score was 33.8 , which was produced by the Japanese-stroke and Chinese-ideograph model; for Chinese-Japanese translation, the best BLEU score was 43.9 , which was produced by the Chinese-ideograph and Japanese-stroke model. 


\begin{tabular}{lccccc}
\hline JP-CN NMT & CN_word & CN_char & CN_bpe & CN_ideograph & CN_stroke \\
\hline JP_word & 29.6 & - & - & 30.8 & 30.3 \\
JP_char & - & 31.6 & - & $32.0^{*}$ & $32.1^{*}$ \\
JP_bpe & - & - & $31.5(31.7)$ & 31.6 & 31.7 \\
JP_ideograph & 30.4 & $33.1^{*}$ & $33.3^{*}$ & $32.0^{*}\left(32.4^{*}\right)$ & $33.4^{*}$ \\
JP_stroke & 30.3 & $33.4^{*}$ & $32.6^{*}$ & $\mathbf{3 3 . 8}^{*}$ & $32.1^{*}(31.2)$ \\
\hline \hline CN-JP NMT & JP_word & JP_char & JP_bpe & JP_ideograph & JP_stroke \\
\hline CN_word & $40.0(40.0)$ & - & - & 40.5 & 40.1 \\
CN_char & $42.1(40.4)$ & 41.7 & - & $43.1^{*}$ & $42.2^{*}$ \\
CN_bpe & 42.1 & - & $42.0(42.3)$ & $43.1^{*}$ & $42.2^{*}$ \\
CN_ideograph & $43.2^{*}$ & $43.5^{*}$ & $43.0^{*}$ & $42.6^{*}\left(42.7^{*}\right)$ & $\mathbf{4 3 . 9}^{*}$ \\
CN_stroke & $43.0^{*}$ & $43.3^{*}$ & $42.5^{*}$ & $42.9^{*}$ & $42.2^{*}(41.1)$ \\
\hline
\end{tabular}

Table 5: Experimental results (BLEU scores) for Japanese/Chinese NMT systems. The row headers and column headers indicate which source and target data were used in the training. In particular, "word" and "char" are character level data without BPE segmentation, while "bpe" (character level), "ideograph", and "stroke" (subcharacter level) are data with BPE segmentation. The scores in parentheses indicate the models that had a shared vocabulary, whenever applicable. The italic numbers represent the two Pinyin baselines used for comparative purposes, namely the "WdPyT" model, which uses Pinyin words with tones as the source data, and the "factoredNMT" model, which uses Pinyin characters as factors (Du and Way, 2017). Note that these two baselines can only have Chinese data on the encoder side. The $*$ superscripts indicate that a score is significantly better than the best baseline result.

\section{Discussions}

\subsection{Translation Examples}

Table 6 shows some of the translation examples. There is a rare proper noun “松下電器 (Matsushita Electric)" (OOV) in the source sentence. The word baseline model cannot decode this; therefore, an <unk> symbol is produced. The character baseline model avoids the OOV problem. However, the underlined parts in both baseline translations seem to be word-for-word translations from the Japanese source sentence (“松下 電器 グループでは”), which become a prepositional phrase in Chinese (“在 松下电器集团中 (in Matsushita Electric Group)"). This makes the translation ungrammatical because there will be no noun phrase as the subject in the sentence. Our best model (i.e., sub-character based NMT model using Japanese stroke data and Chinese ideograph data) can solve these two problems by better encoding the source sentence and can produce translations both without OOV and with a noun phrase as the sentence subject.

\subsection{Strokes vs. Ideographs}

The experimental results show that in NMT models, different logographic languages appear to prefer sub-character units with different granularities. A very clear tendency that was observed consistently in both experiments was that ideographs worked better for Chinese and strokes worked better for Japanese. This difference might be because of the differences in the writing systems. In addition to Kanji (Chinese characters), Japanese uses Hiragana and Katakana, which are standalone alphabets.

Moreover, as described in Section 4, stroke models tended to perform more poorly than ideograph models. This probably occurred because to achieve a fair comparison between all baseline models and proposed models, the same hyperparameter configurations were used. For example, the embedding dimensions for all vocabularies were set to 300 . This might be appropriate for vocabularies of character-based data and ideograph data having vocabulary sizes larger than 500. However, the stroke data only has a vocabulary size of approximately 30 , which is too disproportional. This phenomenon might also account for the decrease in BLEU scores when shared vocabularies were applied to stroke models.

\subsection{The Encoding and Decoding Process}

In comparison with character level data, subcharacter level data (such as ideographs and strokes) can be used to generate much smaller and more concentrated vocabularies. This is helpful during both the encoding and decoding processes. Vocabularies constructed using characterlevel data are known to be very skewed, containing both very frequent words and very rare 


\begin{tabular}{|c|c|}
\hline Model & Sentence \\
\hline Source & $\begin{array}{l}\text { 松下 電器 グループ で は, 経営 理念の 基本 として } 1991 \text { 年 に「環境 } \\
\text { 宣言」をを制定し た。 }\end{array}$ \\
\hline Reference & 作为 经营 理念 , 松下电气集团 于 1991年 制定 了 《环境 宣言 》。 \\
\hline $\begin{array}{l}\text { Baseline } \\
\text { (Word) }\end{array}$ & 在 <unk> 集团中，1991年制定了“环境 宣言” 作为 经营 理念的基础。 \\
\hline $\begin{array}{l}\text { Baseline } \\
\text { (Char) }\end{array}$ & $\begin{array}{l}\text { 在 松下电器 集团 中，作为 经营 理念 的 基础，1991年 制定了《环境 } \\
\text { 宣言 》。 }\end{array}$ \\
\hline $\begin{array}{l}\text { Best Model } \\
\text { (JP-stroke- } \\
\text { CN-ideograph) }\end{array}$ & 松下电器集团，作为 经营 理念 的 基础，1991年 制定 了“环境 宣言”。 \\
\hline $\begin{array}{l}\text { English } \\
\text { Translation }\end{array}$ & $\begin{array}{l}\text { The Matsushita Electric Group enacted the "Environmental Declaration" as the basis of } \\
\text { its business philosophy in } 1991 .\end{array}$ \\
\hline
\end{tabular}

Table 6: Translation examples of Japanese-Chinese NMT systems. Note that “松下电器” as a proper noun, could be handled properly in sub-character based translation systems.

words. As a result, during training, the neurons responsible for high-frequency words might be updated many times, while the neurons responsible for low-frequency words might be updated only a very limited number of times. This will potentially harm translation performance for lowfrequency words.

However, this problem can be alleviated by applying sub-character units. Because ideographs and strokes are repeatedly shared by different characters, no items occur with very low frequencies. More instances can be found in the training data, even for the least frequent sub-character items. Therefore, the translation performance for low-frequency items could be much better.

\section{Conclusions and Future Work}

This study was the first attempt to use subcharacter units in NMT models. Our results not only confirmed the positive effects of using ideograph and stroke sequences in NMT tasks, but also indicated that different logographic languages actually preferred different sub-character granularities (namely, ideograph for Chinese and stroke for Japanese). Finally, this paper presented a simple method for extending the available corpus from the character level to the sub-character level. During this process, we maintained a oneto-one relationship between the original characters and transformed sub-character sequences. As a result, this simple and straightforward method achieved consistently better results for NMT systems used to translate logographic languages, and could be easily applied to similar scenarios.
Many questions remain to be answered in future work. The first question involves the relative benefits of ideograph data and stroke data, and the effects of shared vocabularies. We have not yet explained why there are considerable differences in performance. In particular, for NMT models in which both sides have stroke data, why does performance drop dramatically when shared vocabularies are applied? We discussed the possible reasons for this phenomenon in Section 5.2; however, further investigation is needed.

Another important issue is as follows: when characters are transformed into ideographs and strokes, no structural information is considered. This causes repetitions in data, and we must add tags at the end of each sequence to differentiate them. A better way to solve this problem would be to have structural information directly encoded in the data.

\section{Acknowledgments}

This work was partially supported by JSPS Grantin-Aid for Young Scientists (B) Grant Number JP16K16117.

\section{References}

Dzmitry Bahdanau, Kyunghyun Cho, and Yoshua Bengio. 2015. Neural machine translation by jointly learning to align and translate. In ICLR 2015.

Shaosheng Cao, Wei Lu, Jun Zhou, and Xiaolong Li. 2017. Investigating stroke-level information for learning Chinese word embeddings. In ISWC-16. 
Kyunghyun Cho, Bart van Merrienboer, Caglar Gulcehre, Dzmitry Bahdanau, Fethi Bougares, Holger Schwenk, and Yoshua Bengio. 2014. Learning phrase representations using RNN encoder-decoder for statistical machine translation. In EMNLP 2014, pages 1724-1734.

Jinhua Du and Andy Way. 2017. Pinyin as subword unit for Chinese-sourced neural machine translation. In Irish Conference on Artificial Intelligence and Cognitive Science, page 11.

Sepp Hochreiter and Jbrgen Schmidhuber. 1997. Long short-term memory. Neural computation, 9(8):1735-1780.

Philipp Koehn. 2004. Statistical significance tests for machine translation evaluation. In EMNLP 2014.

Shaohui Kuang and Lifeng Han. 2018. Apply Chinese radicals into neural machine translation: Deeper than character level. In 30Th European Summer School In Logic, Language And Information.

Taku Kudo. 2018. Subword regularization: Improving neural network translation models with multiple subword candidates. In ACL 2018.

Frederick Liu, Han Lu, Chieh Lo, and Graham Neubig. 2017. Learning character-level compositionality with visual features. In $A C L$ 2017, pages 2059-2068.

Thang Luong, Hieu Pham, and Christopher D Manning. 2015. Effective approaches to attention-based neural machine translation. In EMNLP 2015, pages $1412-1421$.

Toshiaki Nakazawa, Manabu Yaguchi, Kiyotaka Uchimoto, Masao Utiyama, Eiichiro Sumita, Sadao Kurohashi, and Hitoshi Isahara. 2016. ASPEC: Asian scientific paper excerpt corpus. In LREC-9, pages 2204-2208.

Graham Neubig. 2013. Travatar: A forest-to-string machine translation engine based on tree transducers. In ACL 2013: System Demonstrations, pages 91-96.

Haiyun Peng, Erik Cambria, and Xiaomei Zou. 2017. Radical-based hierarchical embeddings for Chinese sentiment analysis at sentence level. In FLAIRS30 .

Rico Sennrich, Barry Haddow, and Alexandra Birch. 2016. Neural machine translation of rare words with subword units. In $A C L$ 2016, pages 1715-1725.

Xinlei Shi, Junjie Zhai, Xudong Yang, Zehua Xie, and Chao Liu. 2015. Radical embedding: Delving deeper to Chinese radicals. In ACL-IJCNLP 2015, pages 594-598.

Ilya Sutskever, Oriol Vinyals, and Quoc V. Le. 2014. Sequence to sequence learning with neural networks. In NIPS, pages 3104-3112.
Yota Toyama, Makoto Miwa, and Yutaka Sasaki. 2017. Utilizing visual forms of Japanese characters for neural review classification. In IJCNLP 2017, pages 378-382.

Ashish Vaswani, Noam Shazeer, Niki Parmar, Jakob Uszkoreit, Llion Jones, Aidan N. Gomez, Lukasz Kaiser, and Illia Polosukhin. 2017. Attention is all you need. In NIPS 2017, pages 6000-6010.

Yining Wang, Long Zhou, Jiajun Zhang, and Chengqing Zong. 2017. Word, subword or character? an empirical study of granularity in ChineseEnglish NMT. In China Workshop on Machine Translation, pages 30-42. Springer.

Yonghui Wu, Mike Schuster, Zhifeng Chen, Quoc V. Le, Mohammad Norouzi, Wolfgang Macherey, Maxim Krikun, Yuan Cao, Qin Gao, Klaus Macherey, Jeff Klingner, Apurva Shah, Melvin Johnson, Xiaobing Liu, ukasz Kaiser, Stephan Gouws, Yoshikiyo Kato, Taku Kudo, Hideto Kazawa, Keith Stevens, George Kurian, Nishant Patil, Wei Wang, Cliff Young, Jason Smith, Jason Riesa, Alex Rudnick, Oriol Vinyals, Greg Corrado, Macduff Hughes, and Jeffrey Dean. 2016. Google's neural machine translation system: Bridging the gap between human and machine translation. arXiv:1609.08144 [cs].

\section{Appendix A Strokes in CNS11643 Charset}

\begin{tabular}{|c|c|}
\hline Type & Strokes \\
\hline Horizontal & - \\
\hline Vertical & 1 \\
\hline Left-falling & , J \\
\hline Right-falling & $\cdot \backslash \backslash$ \\
\hline Break & 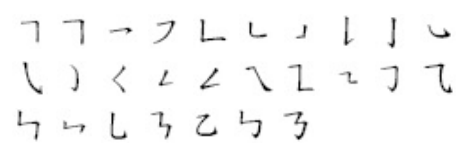 \\
\hline
\end{tabular}

